\title{
Combined Treatment with Lymphaticovenular Anastomosis and Ethanol Sclerotherapy for Cystic Lymphatic Malformation Affecting a Limb
}

Shuhei Yoshida ${ }^{1}$, Hirofumi Imai ${ }^{1}$, Solji Roh ${ }^{1}$, Toshiro Mese ${ }^{1}$, and Isao Koshima ${ }^{1}$

${ }^{1}$ Hiroshima University Hospital

March 1, 2022

\begin{abstract}
This case series describes use of a combination of Lymphaticovenular anastomosis (LVA) and sclerotherapy to treat cystic lymphatic malformations with satisfactory results. In the first case, the patient presented with persistent lymphocele after resection surgery. Our second and third cases presented with cystic lymphangioma in the subclavicular and thigh regions.
\end{abstract}

TITLE

Combined Treatment with Lymphaticovenular Anastomosis and Ethanol Sclerotherapy for Cystic Lymphatic Malformation Affecting a Limb

Authors:

Shuhei Yoshida, M.D, Ph. D [1]., Hirofumi Imai, M.D[1]., Solji Roh, M.D[1]., Toshiro Mese, M.D[1]., Isao Koshima, M.D, Ph. D [1].

All authors' full affiliation;

The International Center for Lymphedema, Plastic and reconstructive Surgery, Hiroshima University Hospital [1].

Corresponding Author; Shuhei Yoshida,

Email: syuheiyoshida44@gmail.com

ZIP 734-8551

1-2-3, Kasumi, Minami-ku, Hiroshima, JAPAN

TEL/FAX: +81 82-257-5555/+81 82-257-5851

Keywords; cystic lymphangioma; sclerotherapy; lymphaticovenular anastomosis; lymphedema. Declaration of conflicting interests

The author(s) declared no potential conflicts of interest with respect

to the research, authorship, and/or publication of this article.

Funding

The author(s) received no financial support for the research, authorship, and/or publication of this article. 


\title{
A list of abbreviations
}

\section{LVA; Lymphaticovenular anastomosis}

\section{ICG; Indocyanine green}

\section{UEL index; Upper extremity lymphedema index}

\author{
MRI; Magnetic Resonance Imaging
}

\section{CT; Computed Tomography}

\section{INTRODUCTION}

Cystic lymphangioma originates in the embryonic stage of life and leads to failure of communication and lymph drainage into the venous system $(1,2)$. There is no consensus on its management, which includes observation, aspiration, injection, cryotherapy, electrocautery, radiation, laser therapy, ligation, and excision depending on the size of the lesion, its anatomic site, and complications (3). Cystic lymphangioma can be treated effectively with surgery or sclerotherapy, but further investigation is necessary to determine which is superior (4).

A lymphocele can develop after lymph node dissection, kidney transplantation, or oncologic surgery (57), and if large enough, may result in pain, infection, and lower extremity lymphedema (8-11). Several treatment options are available, but there is no consensus as to which is the most effective. Needle aspiration and percutaneous catheter drainage, which are commonly used in the initial management of symptomatic lymphocele, have had initial cure rates of up to $80 \%$, but treated lymphoceles are complicated by infection in up to $50 \%$ of patients, with recurrence in $80-90 \%$ of cases $(8,9,12)$. Sclerotherapy has been reported to have success rates of $77 \%$ and $98 \%$ that were inversely proportional to the size of the lymphocele $(8,12)$.

Although the pathogenesis of cystic lymphangioma is different from that of lymphocele, the main pathophysiological feature, namely, a cystic lymphatic malformation connecting to the lymphatic vessels, and the treatment are the same. Sclerotherapy is an effective treatment option for both types of lymphatic malformation. However, sclerosants cause fibrosis and obliteration of both a lymphocele and a ruptured lymphatic vessel (13), thereby impairing lymphatic drainage, which increases the risk of lymphedema (1416). Lymphaticovenular anastomosis (LVA) recently garnered attention in the field of microsurgery as a minimally invasive or prophylactic treatment for lymphedema (17-22) and cystic lymphatic malformation (23-28), although its success rate and indications in terms of the size of the lymphocele for which it is most effective remain unclear. Some reports have described combined treatment for lymphocele consisting of surgical excision and LVA for large or persistent lymphoceles $(29,30)$.

Combined treatment using LVA with sclerotherapy may be an alternative minimally invasive treatment option. In this report, we describe three patients with lymphocele or cystic lymphangioma in the extremities whom we have treated using LVA and ethanol sclerotherapy with satisfactory results.

\section{MATERIAL AND METHODS}

This study was approved by the Ethics Committee of Hiroshima University Hospital. Written informed consent was obtained from each patient. The procedure for LVA combined with sclerotherapy is described below. Before surgery, indocyanine green (ICG) lymphography is performed. First, $0.2 \mathrm{~mL}$ of ICG (Diagnogreen 0.25\%; Daiichi Sankyo, Tokyo, Japan) is injected subcutaneously into both lower extremities at the first web space of the foot and the lateral border of the Achilles tendon or into both upper extremities at the first webspace of the hand and the palmar side of ulnar wrist, depending on which limb is affected. Circumferential fluorescent images of the lymphatic drainage channels are then obtained using an infrared camera system (Photodynamic Eye; Hamamatsu Photonics K.K., Hamamatsu, Japan). All lymphatic pathways and areas of stasis are marked on the skin of each affected extremity.

The lower or upper extremity lymphedema index (LEL or UEL index) is calculated as follows: (sum of the squares of the circumferences at 5 areas in lower or upper extremity)/(body mass index) (31). 
Combined treatment with LVA and ethanol sclerotherapy is performed as follows. Ethanol sclerotherapy is performed first. All the fluid in the cavity is initially evacuated via a small skin incision (Supplemental Digital Content, Video 1). The lymphocele is then catheterized using a round-type, $15 \mathrm{Fr}$, 5-mm diameter Blake@ silicone drain, (Johnson \& Johnson K.K., Tokyo, Japan). Next, absolute ethanol is instilled to fill quarter to half of the estimated volume of the cavity and left in situ for $20 \mathrm{~min}$. During this time, care is taken to ensure complete exposure of the entire surface area of the lymphocele to the sclerosant. All the fluid is then discharged and saline is instilled up to one-half of the estimated volume of the cavity. Next, all the fluid is discharged again. The procedure is repeated 5 times, after which the catheter is left in place for drainage.

LVA is performed soon after ethanol sclerotherapy. Incisions are made along the marked linear pattern or great saphenous vein where the linear pattern is undetectable because of dermal backflow. A 2-3-cm skin incision is made and the collecting lymphatics and subcutaneous veins are dissected and identified under a microscope. If no signs of venous insufficiency or hypertension are observed in the subcutaneous vein intraoperatively, the vein and lymphatic vessels are anastomosed in an end-to-end manner using 11-0 or 12-0 nylon suture under a microscope. Postoperatively, patency is confirmed by backflow of blood into the lymphatics and lymph flow into the veins by massaging the peripheral region. All LVAs are created between the distal lymphatics and the proximal veins in an end-to-end manner. The silicone drain is left in place until the daily drainage volume has dropped below $50 \mathrm{~mL}$. The patient remains in the hospital until the drain is removed. Prophylactic antibiotics are administered for 1 week. Compression therapy is started immediately after surgery using a cotton bandage. A Class 1 compression garment is applied after discharge. Compression therapy is stopped 6 months after surgery.

\section{Case 1: persistent lymphocele after resection of soft tissue sarcoma}

The patient was a 49-year-old man who was referred to our institution with a refractory lymphocele in the lateral aspect of the left thigh. He had undergone wide resection of an extraskeletal myxoid chondrosarcoma $(11 \times 8 \times 13 \mathrm{~cm})$, including parts of the rectus femoris, gluteus medius, sartorius, and vastus lateralis, as well as the skin overlying the tumor 2 months before being referred to our department (Fig. 1a, 1b). Percutaneous needle aspiration had been performed under ultrasonographic guidance once weekly, and 2000-3000 $\mathrm{mL}$ of fluid was aspirated each time (Supplemental video 1). The volume of the lymphocele was estimated to be 2000-3000 mL No signs of infection had been observed despite the repeated percutaneous aspiration. He had also received conventional compression therapy using a compression garment (Cotton, Unisex, Thigh-high, CCL1; Sigvaris, Winterthur, Switzerland). However, there was no decrease in the volume of the lymphocele. The lymphocele measured $25 \times 16 \times 12 \mathrm{~cm}$ and extended from the middle of the thigh to the anterior superior iliac spine in the lateral aspect of the thigh. The depth of the lymphocele was between the subcutaneous fat layer and the muscle layer.

ICG lymphography revealed signs of dermal backflow of lymph in the proximal area of the anteromedial and inguinal areas of the left thigh (Fig. 1c). We could not detect the actual lymphatic routes flowing into the lymphocele because of the dermal backflow in the thigh area. There was no history of heart or kidney failure, liver cirrhosis, hypoproteinemia, deep vein thrombosis, chronic venous obstruction, thyroid dermopathy, other endocrine cause of edema, or drug-induced edema. The dermal backflow pattern was limited to the left thigh area. There was no family history of lymphedema. Based on these findings, we made a diagnosis of left LEL secondary to resection of soft tissue sarcoma with lymphocele.

We elected to perform combined treatment with LVA and ethanol sclerotherapy to treat the lymphocele and reduce the risk of progression of lymphedema. In this case, the LEL index was 306. Surgery was performed under spinal anesthesia., with $150 \mathrm{~mL}$ of absolute ethanol instilled each time. In total, 6 LVAs were created at peripheral sites extending from the lymphocele in the left lower extremity (Fig. 2a, 2b). The total operation time was 4 hours. The postoperative course was uneventful. There was a gradual decrease in the drainage volume (Fig. 3). The drain was removed on postoperative day 14.

One year later, the LEL index was 270 in the left lower extremity. The lymphocele resolved no recurrence 
was noted during a follow-up period of 12 months (Fig. 4a, 4b). Postoperative ICG lymphography performed at this time revealed reduced dermal backflow of lymph in the left thigh (Fig. 4c).

\section{Case 2: Cystic lymphangioma extending from the axilla to the subclavicular area}

The patient was an 18-year-old woman who was referred to our institution with a soft mass extending from the axilla to the anterior chest wall which she had noticed 1 month earlier (Fig. 5a). Magnetic resonance imaging and computed tomography revealed a lymphangioma with a diameter of $12 \mathrm{~cm}$ and a maximum depth of $8 \mathrm{~cm}$ extending from the axilla to the subclavicular area beneath pectoralis major and pectoralis minor (Fig. 5b, 5c).

Combined treatment of LVA with sclerotherapy was planned and performed under general anesthesia. The UEL index was 110. ICG lymphography revealed a linear pattern along the ulnar side of the right upper limb (Fig. 6a). Absolute ethanol at a volume of $50 \mathrm{~mL}$ was instilled each time. Two LVAs were created at peripheral sites extending from the cyst of the right upper arm (Fig. 6b).

The postoperative course was uneventful. The drainage volume decreased rapidly. The drain was removed on postoperative day 4. One year later, the UEL index was 108 in the left upper extremity. The cystic lesion resolved and no recurrence was noted during a follow-up period of 18 months (Fig. 7a, 7b).

\section{Case 3: Cystic lymphangioma in thigh area}

The patient was a 60-year-old man who was referred to our institution with a 1-year history of right knee swelling (Fig. 8a). Magnetic resonance imaging revealed a lymphangioma of $16 \mathrm{~cm}$ in diameter in the thigh region above the knee (Fig. 8 b).

Combined treatment using LVA with sclerotherapy was planned and performed under general anesthesia. The LEL index before surgery was 343. ICG lymphography revealed a linear pattern along the great saphenous vein (Fig. 8c). Ethanol sclerotherapy was performed using $20 \mathrm{~mL}$ absolute ethanol each time. Four LVAs were established in the right leg (Fig. 9). The postoperative course was uneventful. Drainage volume decreased quickly. The drain was removed 7 days postoperatively.

At 2 years after surgery, the LEL index was 343 in the right lower extremity. The cystic lymphoma resolved and no recurrence was noted during follow-up (Fig. 10a, 10b).

\section{DISCUSSION}

Cystic lymphangioma is rarely encountered in adults. Its etiology remains unclear, but it is presumed to be congenital or a result of obstruction and retention of lymph fluid in developing lymphatic vessels (32). Subcutaneous lymphocele, particularly at the site of a surgical scar, is caused by a lesion in a lymphatic vessel during a surgical procedure, with continued lymph flow within the cavity under the scar. Combined aspiration and compression are not always effective, and there is a high rate of recurrence.

These two types of lymphatic malformation have different pathogenetic mechanisms. However, the main pathophysiological feature, namely, the cystic lymphatic malformation connecting to the lymphatic vessels, and the treatment are common to the two conditions. Sclerotherapy is an effective option for both types of lymphatic malformation. Sclerosants cause fibrosis and obliteration of both a lymphocele and a ruptured lymphatic vessel (13), thereby impairing lymphatic drainage. The same effect is seen when performing macroscopic ligation of subcutaneous tissue containing lymphatic vessels. We believe that the ideal treatment in these cases is reconstruction to restore lymphatic flow by anastomosing a ruptured lymphatic vessel to another lymphatic vessel or to a vein to stop the lymph flow within the cyst and maintain effective lymph drainage. LVA is an excellent option for the treatment of lymphocele or cystic lymphangioma when all the lymphatics flowing into the cyst are detectable. However, they are not always detectable. especially for cystic lymphatic malformations that are large, persistent, or infected. In such cases, the lymphatics are already compromised and undetectable, so, LVA alone is not sufficient to treat cystic lymphatic malformations in some cases. This LVA procedure is particularly indicated for mild-to-moderate conditions. For smaller volumes of accumulated fluid, creating 1 or more LVAs is sufficient, but with larger volumes this becomes 
inadequate due to the large residual dead space inside the capsule, which tends to fill spontaneously with interstitial fluid. Consequently, we integrated the lymphatic treatment with sclerotherapy, and thus were able to adequately close the dead space using sclerotherapy. Surgical excision of cystic lymphatic malformations or filling the dead space with a muscle flap has been proposed as a form of combined LVA treatment for cystic lymphatic malformations $(29,30)$. While these methods are necessary in some cases, they are relatively more invasive than sclerotherapy with LVA. The main function of sclerotherapy is to close the residual dead space and prevent formation of an empty space in which fluid can easily accumulate. The LVAs are established primarily for diverting lymph flow and preventing recurrence of the primary disease.

The relationship between risk of lymphedema and cystic lymphatic malformations is controversial. Some studies have found no significant evidence to suggest that the presence of pelvic cystic lymphatic malformations causes lower limb lymphedema (33-37). In contrast, cystic lymphatic malformations have been reported to be an independent risk factor for lymphedema (14-16,38). Moreover, surgical treatment can involve repeat exploration of the cystic lymphatic malformation and ligation of subcutaneous tissue at presumed sites of lymphatic vessels without direct microscopic visualization. Therefore, both sclerotherapy and ligation can definitively block lymph flow, implying a risk of lymphedema (39-41).

Nevertheless, there are concerns that treatment of a cystic lymphatic malformation alone leads to worsening of lymphedema or increases the risk of its development. LVA, on the other hand, provides favorable results, not only in prevention but also in treatment of peripheral lymphedema (42-44). Recently, the focus of lymphedema treatment has shifted to risk reduction and prevention (45). Therefore, it is desirable to perform LVA combined with sclerotherapy in terms of preventing lymphedema and treating cystic lymphatic malformations. It is expected that combined treatment using LVA with sclerotherapy will offer a complementary minimally invasive treatment option for a large or persistent cystic lymphatic malformation.

\section{Conclusion}

LVA is thought to be an excellent less invasive treatment option when the lymphatics flowing into the cyst are detectable or can be targeted. However, this is not always the case, especially for a cyst that is large, persistent, or infected. For this reason, we combined LVA with sclerotherapy. This allowed for a relatively less invasive method with closure of the dead space using sclerotherapy without further exacerbation or occurrence of lymphedema. Hence, combined treatment using LVA with sclerotherapy can be a complementary minimally invasive treatment option for a large or persistent lymphocele.

\section{References}

1. Damaskos C, Garmpis N, Manousi M, Garmpi A, Margonis GA, Spartalis E, Doula C, Michail-Strantzia C, Patelis N, Schizas D, Arkoumanis PT, Andreatos N, Tsourouflis G, Zavras N, Markatos K, Kontzoglou K, Antoniou EA. Cystic hygroma of the neck: single center experience and literature review. Eur Rev Med Pharmacol Sci. 2017 Nov;21(21):4918-4923.

2. Wiegand S, Eivazi B, Barth PJ, von Rautenfeld DB, Folz BJ, Mandic R, Werner JA. Pathogenesis of lymphangiomas. Virchows Arch. 2008 Jul;453(1):1-8.

3. Ha J, Yu YC, Lannigan F., A review of the management of lymphangiomas. Curr Pediatr Rev. 2014;10(3):238-48.

4. Adams MT, Saltzman B, Perkins JA. Head and neck lymphatic malformation treatment: a systematic review. Otolaryngol Head Neck Surg. 2012 Oct;147(4):627-39.

5. Kissin MV, Simpson DA, Easton D, White H, Westbury G. Prognostic factors related to survival and groin recurrence following therapeutic lymph node dissection for lower limb malignant melanoma. $\mathrm{Br}$ J Surg 1987;74:1023-1026.

6. Manci N, Marchetti C, Esposito F, De Falco C, Bellati F, Giorgini M, Angioli R, Panici PB. Inguinofemoral lymphadenectomy: Randomized trial comparing inguinal skin access above or below the inguinal ligament. Ann Surg Oncol 2009;16:721-728.

7. Pittaluga $\mathrm{P}$, Chastanet S. Lymphatic complications after varicose veins surgery: Risk factors and how to avoid them. Phlebology 2012;27(Suppl 1):139-142. 
8. Mahrer A, Ramchandani P, Trerotola SO, Shlansky-Goldberg RD, Itkin M. Sclerotherapy in the management of postoperative lymphocele. J Vasc Interv Radiol 2010;21:1050-3.

9. Kim HY, Kim JW, Kim SH, Kim YT, Kim JH. An analysis of the risk factors and management of lymphocele after pelvic lymphadenectomy in patients with gynecologic malignancies. Cancer Res Treat 2004;36:377-83.

10. Tasar M, Gulec B, Saglam M, Yavuz I, Bozlar U, Ugurel S. Posttransplant symptomatic lymphocele treatment with percutaneous drainage and ethanol sclerosis: long-term follow-up. Clin Imaging 2005;29:109-16.

11. Gallotta V, Fanfani F, Rossitto C, Vizzielli G, Testa A, Scambia G, et al. A randomized study comparing the use of the Ligaclip with bipolar energy to prevent lymphocele during laparoscopic pelvic lymphadenectomy for gynecologic cancer. Am J Obstet Gynecol 2010;203:483.e1-6.

12. Klode J, Klötgen K, Körber A, Schadendorf D, Dissemond J. Polidocanol foam sclerotherapy is a new and effective treatment for post-operative lymphorrhea and lymphocele. J Eur Acad Dermatol Venereol 2010; 24: 904-9.

13. Chen WL, Huang ZQ, Chai Q, Zhang DM, Wang YY, Wang HJ, Wang L, Fan S. Percutaneous sclerotherapy of massive macrocystic lymphatic malformations of the face and neck using fibrin glue with OK-432 and bleomycin. Int J Oral Maxillofac Surg 2011;40:572-576.

14. S K Bains, N J London. Unilateral lower limb swelling secondary to cavernous lymphangioma. Eur J Vasc Endovasc Surg. 2008;35(3):373-4.

15. A Hadj-Henni, V Ladan-Marcus, I Javerliat, N Bouhzam, L Fouilhe, C Marcus, A Long. Cystic lymphangioma: an unusual cause of lower limb lymphedema. J Mal Vasc. 2008;33(3):155-60.

16. M Saijo, I R Munro, K Mancer. Lymphangioma. A long-term follow-up study. Plast Reconstr Surg. 1975;56(6):642-51.

17. Yoshida S, Koshima I, Imai H, Uchiki T, Sasaki A, Fujioka Y, Nagamatsu S, Yokota K, Yamashita S. Lymphovenous Anastomosis for Morbidly Obese Patients with Lymphedema. Plast Reconstr Surg Glob Open. 2020 May 27;8(5):e2860.

18. Yoshida S, Koshima I, Imai H, Uchiki T, Sasaki A, Fujioka Y, Nagamatsu S, Yokota K, Harima M, Yamashita S, Tashiro K. Characteristics and outcomes of lymphaticovenular anastomosis in older patients with bilateral involvement versus younger patients with unilateral involvement in lower extremity lymphedema. J Vasc Surg Venous Lymphat Disord. 2020 Jul;8(4):646-657.

19. Yoshida S, Koshima I, Imai H, Uchiki T, Sasaki A, Fujioka Y, Nagamatsu S, Yokota K. Lymphaticovenous Anastomosis in Fowler's Position under Local Anesthesia. Plast Reconstr Surg Glob Open. 2020 Aug 17;8(8):e3067.

20. Yoshida S, Koshima I, Hamada Y, Sasaki A, Fujioka Y, Nagamatsu S, Yokota K, Harima M, Yamashita S. Lymphovenous Anastomosis Aids Wound Healing in Lymphedema: Relationship Between Lymphedema and Delayed Wound Healing from a View of Immune Mechanisms. Adv Wound Care (New Rochelle). 2019 Jun 1;8(6):263-269.

21. Yamamoto T, Yamamoto N, Yamashita M, Furuya M, Hayashi A, Koshima I. Efferent Lymphatic Vessel Anastomosis: Supermicrosurgical Efferent Lymphatic Vessel-to-Venous Anastomosis for the Prophylactic Treatment of Subclinical Lymphedema. Ann Plast Surg. 2016 Apr;76(4):424-7.

22. Agarwal S, Garza RM, Chang DW. Lymphatic Microsurgical Preventive Healing Approach (LYMPHA) for the prevention of secondary lymphedema. Breast J. 2020 Apr;26(4):721-724.

23. Ayestaray B, Esnault M, Godard M, Picquot S. Treatment of refractory groin lymphocele by surrounding supermicrosurgical lymphaticovenous anastomosis. Arch Plast Surg. 2018 May;45(3):290-291.

24. Giacalone G, Yamamoto T, Hayashi A, Belva F, Gysen M, Hayashi N, Yamamoto N, Koshima I. Lymphatic supermicrosurgery for the treatment of recurrent lymphocele and severe lymphorrhea. Microsurgery. 2019 May;39(4):326-331.

25. Todokoro T, Furniss D, Oda K, Kawana K, Narushima M, Mihara M, Kikuchi K, Hara H, Yano T, Koshima I. Effective treatment of pelvic lymphocele by lymphaticovenular anastomosis. Gynecol Oncol. 2013 Feb;128(2):209-14.

26. Mihara M, Hayashi Y, Hara H, Todokoro T, Koshima I, Murai N. Lymphatic-venous anastomosis for 
the radical cure of a large pelvic lymphocyst. J Minim Invasive Gynecol. 2012 Jan-Feb;19(1):125-7.

27. Furuse K, Kato M, Morishita Y, Kumagai T, Nakatsukasa S, Kuwata T. Lymphatic Malformation Treated with Lymphatic Malformation-Venous Anastomosis Under Local Anesthesia. Plast Reconstr Surg Glob Open. 2020 Jul 24;8(7):e2974.

28. Kato M, Watanabe S, Watanabe A, Iida T. Flow-oriented Venous Anastomosis to Control Lymph Flow of Lymphatic Malformation. Plast Reconstr Surg Glob Open. 2019 Jul 29;7(7):e2199.

29. Boccardo F, Dessalvi S, Campisi C, Molinari L, Spinaci S, Talamo G, Campisi C. Microsurgery for Groin Lymphocele and Lymphedema After Oncologic Surgery. Microsurgery. 2014 Jan;34(1):10-3.

30. Scaglioni MF, Meroni M, Fritsche E. Lymphovenous anastomosis (LVA) for treatment of iatrogenic lymphocele in the thigh. Microsurgery. 2020 Apr 28:e30594.

31. Yamamoto T, Matsuda N, Todokoro T, Yoshimatsu H, Narushima M, Mihara M, et al. Lower extremity lymphedema(LEL) index: a simple method for severity evaluation of lower extremity lymphedema. Ann Plast Surg. 2011;67:637-640.

32. Walter C, Valerio F, Aleksandr K, Marta Z, Fabrizio B, Roberto B. Cystic lymphangioma in adult: a case report and a review of the literature. Journal of Surgical Case Reports, 2020;7, 1-3.

33. May K, Bryant A, Dickinson HO, et al. Lymphadenectomy for the management of endometrial cancer. Cochrane Database Syst Rev 2010; CD007585.

34. Biglia N, Librino A, Ottino MC, et al. Lower limb lymphedema and neurological complications after lymphadenectomy for gynecological cancer. Int J Gynecol Cancer 2015;25:521-5.

35. Achouri A, Huchon C, Bats AS, et al. Complications of lymphadenectomy for gynecologic cancer. Eur J Surg Oncol 2013;39:81-6.

36. Lv S, Wang Q, Zhao $\mathrm{W}$, et al. A review of the postoperative lymphatic leakage. Oncotarget 2017;8:69062-75.

37. Neagoe OC, Ionica M, Mazilu O. The Role of Pelvic Lymphocele in the Development of Early Postoperative Complications. Medicine (Baltimore). 2018 Sep;97(37):e12353.

38. Kuroda K, Yamamoto Y, Yanagisawa M, et al. Risk factors and a prediction model for lower limb lymphedema following lymphadenectomy in gynecologic cancer: a hospital-based retrospective cohort study. BMC Womens Health 2017;17:50.

39. Roberts JR, Walters GK, Zenilman ME, Jones CE. Groin lymphorrhea complicating revascularization involving the femoral vessels. Am J Surg 1993;165:341-344.

40. Van den Brande P, von Kemp K, Aerden D, Debing E, Vanhulle A, Staelens I, Haentjens P. Treatment of lymphocutaneous fistulas after vascular procedures of the lower limb: Accurate wound reclosure and 3 weeks of consistent and continuing drainage. Ann Vasc Surg 2012; 26:833-838.

41. Campisi CC, Boccardo F, Piazza C, Campisi C. Evolution of chylous fistula management after neck dissection. Curr Opin Otolaryngol Head Neck Surg 2013;21:150-156.

42. Auba C, Marre D, Rodraguez-Losada G, Hontanilla B. Lymphaticovenular anastomoses for lymphedema treatment: 18 months postoperative outcomes. Microsurgery 2012;32(4):261-268.

43. Felmerer G, Sattler T, Lohrmann C, Tobbia D. Treatment of various secondary lymphedema by microsurgical lymph vessel transplantation. Microsurgery 2012;32(3):171-177.

44. Campisi C, C Bellini, C Campisi, Accogli S, Bonioli E, Boccardo F. Microsurgery for lymphedema: Clinical research and long-term results. Microsurgery 2010;30(4):256-260.

45. Jørgensen MG, Toyserkani NM, Sørensen JA. The effect of prophylactic lymphovenous anastomosis and shunts for preventing cancer-related lymphedema: a systematic review and meta-analysis. Microsurgery 2018;38:576-85.

\section{Legends}

Supplemental Video 1 All fluid in the cavity was initially evacuated via a small skin incision.

Figure 1a The patient had undergone wide resection of extraskeletal myxoid chondrosarcoma $(11 \times 8 \times 13$ $\mathrm{cm}$ ) including part of rectus femoris, gluteus medius, sartorius, and vastus lateralis, as well as the skin overlying the tumor 2 months before referral to our department. 
Figure $1 \mathrm{~b}$ The lymphocele measured $25 \times 16 \times 12 \mathrm{~cm}$ and extended from the middle portion of the thigh to the anterior superior iliac spine in the lateral aspect of the thigh. Percutaneous needle aspiration had been performed once weekly, and 2000-3000 mL fluid had been aspirated on each occasion.

Figure 1c Preoperative indocyanine green lymphography showing dermal backflow of lymph in the proximal area of the anteromedial aspect of the left thigh and the inguinal area. We could not detect the lymphatic routes that flowed into the lymphocele clearly because of the dermal backflow in the thigh area.

Figure 2a Veins and lymphatic vessels anastomosed in an end-to-end manner using 11-0 or 12-0 nylon under a microscope.

Figure 2b Six lymphaticovenular anastomoses were created in peripheral sites extending from the lymphocele in the left lower extremity.

Figure 3 The drainage volume decreased gradually. A silicone drainage tube was left in situ until the daily volume drained decreased below $50 \mathrm{~mL}$. The drain tube was removed on postoperative day 14 .

Figure 4a, 4b The lymphocele resolved with no recurrence during 12 months of follow-up.

Figure 4c Postoperative indocyanine green lymphography showing reduced dermal backflow of lymph in the left thigh after a follow-up period of 12 months.

Fig. 5a Cystic lymphangioma extending from the axilla to the subclavicular area in an 18-year-old woman.

Fig. 5b, 5c Magnetic resonance imaging and computed tomography revealed a lymphangioma measuring 12 $\mathrm{cm}$ in diameter with a maximum depth of $8 \mathrm{~cm}$ extending from the axilla to the subclavicular area beneath pectoralis major and pectoralis minor.

Fig. 6a Indocyanine green lymphography revealed a linear pattern along the ulna side of the right upper limb.

Fig. 6b Two lymphaticovenular anastomoses were established in peripheral sites extending from the cyst in the right upper arm.

Fig. 7a b The cyst resolved with no recurrence during 18 months of follow-up.

Fig. 8a Cystic lymphangioma in the thigh area in a 60-year-old man.

Fig. 8b Magnetic resonance imaging revealed that lymphangioma measuring $16 \mathrm{com}$ in diameter in the thigh region above the knee.

Fig. 8c Indocyanine green lymphography revealed a linear pattern along the great saphenous vein.

Fig. 9 Ethanol sclerotherapy was performed using $20 \mathrm{~mL}$ of absolute ethanol each time. Four lymphaticovenular anastomoses were created in the right leg.

Fig. 10a b The cystic lymphoma resolved with no recurrence during follow-up.

\section{Hosted file}

figures for clinical cas repor .pptx available at https://authorea.com/users/462982/articles/ 558228-combined-treatment-with-lymphaticovenular-anastomosis-and-ethanol-sclerotherapy-

for-cystic-lymphatic-malformation-affecting-a-limb 